\title{
Functional genomics indicates yeast requires Golgi/ER transport, chromatin remodeling, and DNA repair for low dose DMSO tolerance
}

\author{
Brandon D. Gaytán, Alex V. Loguinov, Vanessa Y. De La Rosa, Jan-Michael Lerot and Chris D. Vulpe*
}

Department of Nutritional Science and Toxicology, University of California, Berkeley, CA, USA

Edited by:

Michael Aschner, Vanderbilt

University Medical Center, USA

\section{Reviewed by:}

Jonathan Freedman, National

Institute of Environmental Health

Science, USA

Nallasivam Palanisamy, University of

Michigan, USA

*Correspondence:

Chris D. Vulpe, Department of

Nutritional Science and Toxicology,

University of California, Berkeley,

317 Morgan Hall, Berkeley, CA

94720, USA

e-mail:vulpe@berkeley.edu
Dimethyl sulfoxide (DMSO) is frequently utilized as a solvent in toxicological and pharmaceutical investigations. It is therefore important to establish the cellular and molecular targets of DMSO in order to differentiate its intrinsic effects from those elicited by a compound of interest. We performed a genome-wide functional screen in Saccharomyces cerevisiae to identify deletion mutants exhibiting sensitivity to $1 \%$ DMSO, a concentration standard to yeast chemical profiling studies. We report that mutants defective in Golgi/ER transport are sensitive to DMSO, including those lacking components of the conserved oligomeric Golgi (COG) complex. Moreover, strains deleted for members of the SWR1 histone exchange complex are hypersensitive to DMSO, with additional chromatin remodeling mutants displaying a range of growth defects. We also identify DNA repair genes important for DMSO tolerance. Finally, we demonstrate that overexpression of histone H2A.Z, which replaces chromatin-associated histone H2A in a SWR1-catalyzed reaction, confers resistance to DMSO. Many yeast genes described in this study have homologs in more complex organisms, and the data provided is applicable to future investigations into the cellular and molecular mechanisms of DMSO toxicity.

Keywords: DMSO, dimethyl sulfoxide, functional genomics, functional profiling, yeast, chromatin

\section{INTRODUCTION}

The dipolarity and low toxicity of dimethyl sulfoxide (DMSO) make it an unrivaled solvent in the field of toxicology. DMSO elicits numerous cellular effects, demonstrating the capacity to serve as a cryoprotectant, hydroxyl radical scavenger, and inducer of cellular differentiation and fusion (reviewed by $\mathrm{Yu}$ and Quinn, 1994). The pharmacological properties of DMSO have been documented in the treatment of brain edema, amyloidosis, rheumatoid arthritis, and schizophrenia, with infrequently reported systemic toxicities (Santos et al., 2003). The ubiquity of DMSO as a toxicant and drug solvent demands further identification of the cellular and molecular processes it may perturb, primarily to discern whether its effects influence those mediated by a compound of interest.

The unique genetic tools available in the model eukaryote Saccharomyces cerevisiae facilitate investigations into the cellular and molecular mechanisms of chemical resistance. The collection of barcoded yeast deletion mutants (Giaever et al., 2002) can be exploited to conduct functional genomic analyses (otherwise known as functional profiling) for a compound of interest. Pools of mutants are subjected to chemical treatment, and after DNA extraction, the strain-specific barcodes are amplified and hybridized to a microarray. Signal intensities correspond to strain numbers present in the pool after exposure, and indicate how the given insult alters the growth of individual mutants. With a high degree of conservation to more complex organisms (Steinmetz et al., 2002), yeast is an appealing model that can help identify human chemical susceptibility or resistance genes (Jo et al., 2009a; Blackman et al., 2012).

In this study, we utilized a genome-wide functional screen to identify yeast mutants exhibiting sensitivity to the common solvent DMSO. During preparation of this manuscript, a study was published implicating transcriptional control machinery and cell wall integrity as necessary for DMSO tolerance in S. cerevisiae (Zhang et al., 2013). Similarly, our results demonstrate that mutants lacking components of the SWR1 histone exchange complex exhibit hypersensitivity to DMSO. Here we corroborate and extend Zhang et al. (2013) by identifying additional SWR1 and conserved oligomeric Golgi (COG) complex members as required for DMSO resistance. We also provide extensive dose-response data for various deletion strains and present several novel DMSO-sensitive mutants. Finally, we indicate that overexpression of histone H2A.Z can confer DMSO resistance. Many yeast genes identified in this investigation have homologs that may contribute to DMSO response in more complex organisms.

\section{MATERIALS AND METHODS YEAST STRAINS AND CULTURE}

Functional profiling and confirmation analyses utilized the collection of BY4743 non-essential diploid yeast deletion strains (MATa/MATa his $3 \Delta 1 /$ his $3 \Delta 1$ leu2 $\Delta 0 /$ leu $2 \Delta 0$ lys $2 \Delta 0 / L Y S 2$ MET15/met $15 \Delta 0$ ura $\Delta \Delta 0 /$ ura $3 \Delta 0$, Invitrogen). All assays were performed in liquid rich media ( $1 \%$ yeast extract, $2 \%$ peptone, $2 \%$ dextrose, YPD) at $30^{\circ} \mathrm{C}$ with shaking at $200 \mathrm{rpm}$, except 
overexpression experiments, which used liquid rich media containing 2\% galactose and 2\% raffinose (YPGal + Raf). For overexpression analyses, the HTZ1 and ARP6 HIP FlexGene expression vectors were transformed into strains of the BY4743 background.

\section{FUNCTIONAL PROFILING OF THE YEAST GENOME AND OVERENRICHMENT ANALYSES}

Growth of the homozygous diploid deletion pools (4607 mutants in total), DNA extraction, PCR-amplification of strain barcodes, hybridization of Affymetrix TAG4 arrays, and differential strain sensitivity analysis (DSSA) were performed as described (Jo et al., 2009 b). For DSSA, twelve 1\% DMSO replicates were compared to 12 YPD replicates. Data files are available at the Gene Expression Omnibus (GEO) database. Significantly overrepresented Gene Ontology (GO) and MIPS (Munich Information Center for Protein Sequences) categories within the functional profiling data were identified with FunSpec (Robinson et al., 2002), using a $p$-value cutoff of 0.001 and Bonferroni correction.

\section{GROWTH CURVE AND FLOW CYTOMETRY CONFIRMATION ASSAYS}

Growth curve assays were performed as in North et al. (2011), with DMSO (VWR, \#EM-MX1458-6) added to the desired final concentrations at a minimum two technical replicates per dose. Confirmation of growth defects by a flow cytometry based relative growth assay was performed as in Gaytán et al. (2013). Briefly, a culture containing GFP-tagged wildtype and untagged mutant cells was treated with DMSO, and a ratio of growth was calculated for untagged cells in treated versus untreated samples, as compared to the GFP strain. All graphs display the mean and standard error of three independent cultures. Three tests-regular $t$-test, Welch's test $(t$ test modification assuming unequal variances) and Wilcoxon Rank Sum (Mann-Whitney) test-were simultaneously applied to assess how possible violations of the assumptions underlying $t$-test (homoscedasticity and normality) affect statistical inference outcomes for the data. Raw $p$-values for each test statistic were corrected for multiplicity of comparisons using BenjaminiHochberg correction. $P$-values indicated on graphs are derived from regular $t$-tests, with Welch and Wilcoxon Rank Sum test results (which are more robust but more conservative in terms of adjusted $p$-values) usually in agreement with regular $t$-tests (Table S1).

\section{RESULTS \\ FUNCTIONAL PROFILING IN YEAST IDENTIFIES GENES REOUIRED FOR DMSO TOLERANCE}

Following growth of yeast homozygous diploid deletion mutant pools for 15 generations in 1\% DMSO, DSSA identified 40 strains as sensitive to DMSO, as compared to YPD controls (Table 1; Table S2). To identify the biological attributes required for DMSO tolerance, enrichment analyses for the 40 sensitive strains was performed with FunSpec at a corrected $p$-value of 0.001 . The COG complex, as well as its biological functions (cytoplasm to vacuole targeting pathway and intra-Golgi transport), were overrepresented in both GO and MIPS categories (Table 2).

\section{MUTANTS DEFECTIVE IN GOLGI/ER TRANSPORT ARE SENSITIVE TO DMSO}

Overrepresentation analyses suggested that subunits of COG, a protein complex that mediates fusion of transport vesicles to Golgi compartments, were required for DMSO tolerance. Therefore, we performed relative growth assays in which the growth of COG deletion strains was compared to a wild-type GFP-expressing strain in various DMSO concentrations. Deletion of genes encoding any of the four non-essential subunits of COG (COG5, COG6, COG7, and COG8) resulted in dose-dependent sensitivity to DMSO, with statistically significant growth defects observed at DMSO concentrations as low as $0.25 \%$ (Figure 1A). Growth curve assays also confirmed sensitivity of the individual COG deletions under non-competitive conditions (Figure 1B). To identify additional sensitive Golgi/ER transport strains not present in the functional profiling data, we tested the DMSO sensitivity of various mutants displaying synthetic lethality or sickness with at least one COG gene. Analysis of relative growth by flow cytometry found that strains lacking vacuolar SNAREs (vam7 $\Delta$ and gos $1 \Delta$ ) were DMSO-sensitive (Figure 1A). Growth curve experiments were performed as an alternative for strains demonstrating severe fitness defects in the relative growth assay, with mutants defective in retrograde Golgi transport (ricl $\Delta$, $v p s 51 \Delta$, and $v p s 54 \Delta$ ) as well as those deleted for components of the Guided Entry of Tailanchored (GET) Golgi/ER trafficking complex (get $1 \Delta$ and get $2 \Delta$ ) exhibiting dose-dependent DMSO sensitivity (Figure 1B).

\section{CHROMATIN REMODELING MACHINERY IS REOUIRED FOR DMSO TOLERANCE}

The yaf9s strain, which lacks a subunit common to the SWR1 histone exchange and NuA4 histone H4 acetyltransferase complexes, was identified by DSSA as DMSO-sensitive (Table 1) and confirmed by both competitive growth and growth curve assays to exhibit severe DMSO-dependent growth defects (Figures 2A,B). This stark phenotype prompted us to examine all non-essential SWR1 and NuA4 deletions for DMSO sensitivity, as SWR1 and NuA4 complexes cooperate to alter chromatin structure in yeast (reviewed by Lu et al., 2009). Except for $s w c 7 \Delta$, every SWR1 mutant ( $s w r 1 \Delta, s w c 2 \Delta$, swc3 $\Delta$, $\operatorname{swc5} \Delta$, swc6 $\Delta$, $\operatorname{arp} 6 \Delta$, and $b d f 1 \Delta$ ) was confirmed as sensitive to DMSO, with most displaying similar dose-dependent growth inhibition (Figures 2A,B). Moreover, $h t z 1 \Delta$, a strain lacking the histone variant H2A.Z exchanged for histone $\mathrm{H} 2 \mathrm{~A}$ in nucleosomes by the SWR1 complex (Mizuguchi et al., 2004), displayed growth defects in DMSO (Figure 2A). Several, but not all, non-essential NuA4 deletion mutants (eafl $\Delta$, eaf $3 \Delta$, and eaf7 $\Delta$, but not eaf5 $\Delta$ or eaf6 $\Delta$ ) were DMSOsensitive, however, levels of DMSO-mediated growth inhibition did not approach that of the SWR1 mutants (Figure 2A). We tested additional strains exhibiting both (1) defects in histone modification and (2) synthetic lethality or sickness with SWR1 and/or NuA4 genes (Collins et al., 2007; Mitchell et al., 2008; Costanzo et al., 2010; Hoppins et al., 2011). Absence of components of the Set1C histone $\mathrm{H} 3$ methylase $(\operatorname{swd} 1 \Delta, \operatorname{swd} 3 \Delta$, and $\operatorname{spp} 1 \Delta)$, the Set3C histone deacetylase $(\operatorname{set} 3 \Delta$, sif $2 \Delta$, and $\operatorname{hos} 2 \Delta$, but not $\operatorname{snt} 1 \Delta)$, the SAGA 
Table 1 | Fitness scores for deletion strains identified as significantly sensitive to $1 \%$ DMSO during a 15 generation treatment.

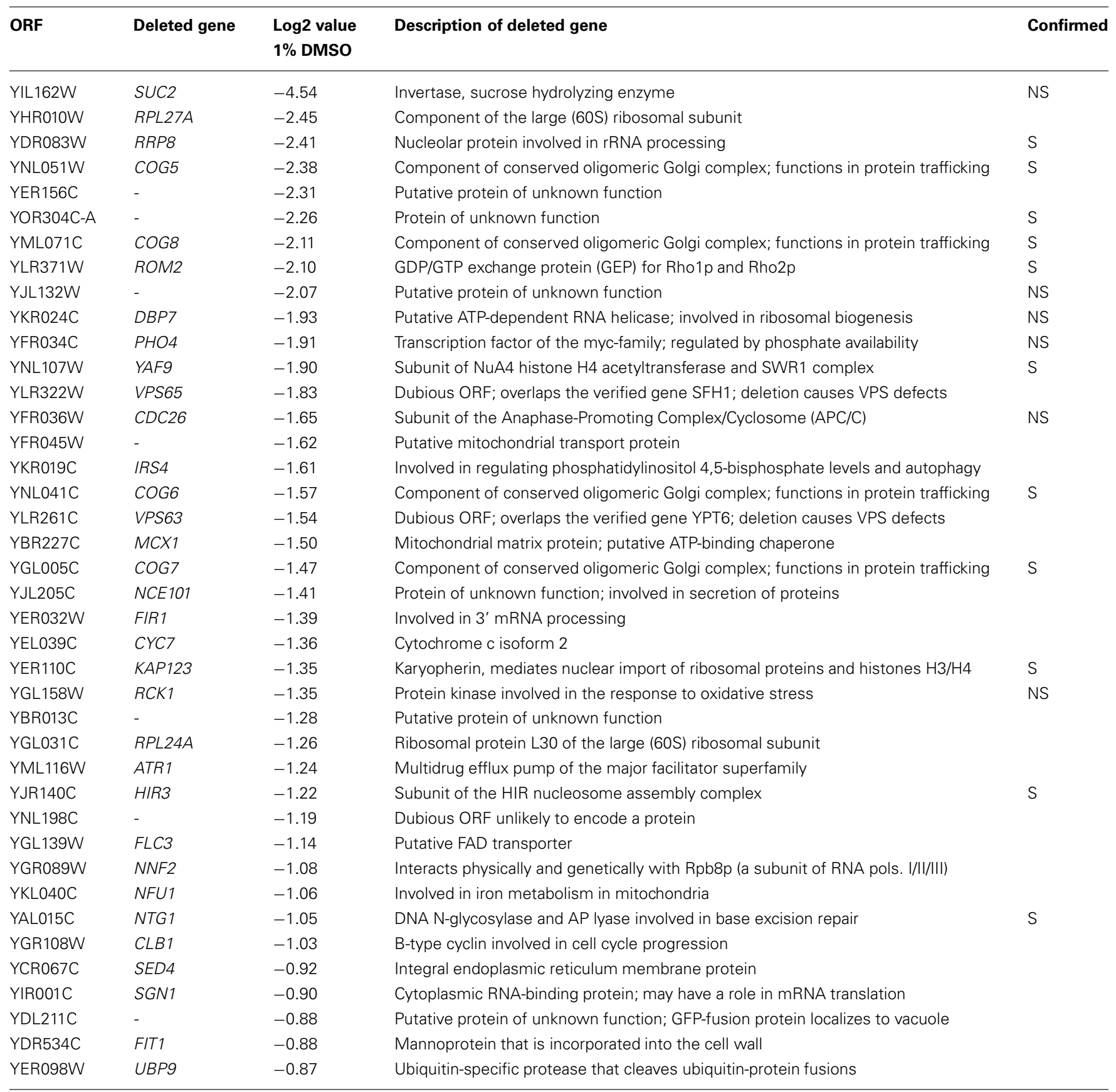

Fitness is defined as the normalized log2 ratio of strain growth in the presence vs. absence of DMSO. The confirmed column indicates whether the strain was confirmed as sensitive (S) or not sensitive (NS) by relative growth assays. Sensitivity is defined as a relative growth ratio of $<0.9$ in DMSO versus a wild-type GFP expressing strain.

acetyltransferase $(g c n 5 \Delta)$ and histone $\mathrm{H} 2 \mathrm{~B}$ deubiquitylation module ( $\operatorname{sgf11\Delta }$ and $u b p 8 \Delta)$, and the Paf1 transcription initiation complex $(c d c 73 \Delta)$ conferred DMSO sensitivity, although none displayed DMSO-mediated growth defects as drastic as SWR1 mutants (Figures 3A-D). DSSA and our relative growth assay identified HIR3, a gene encoding a subunit of the histone regulation (HIR) nucleosome assembly complex, as required for DMSO tolerance, with additional HIR members
(HIR1, HIR2, HPC2) also confirmed as necessary for resistance (Figure 3E).

\section{ADDITIONAL MUTANTS, INCLUDING THOSE INVOLVED IN DNA REPAIR, ARE SENSITIVE TO DMSO}

The NTG1 gene, which encodes a DNA N-glycosylase and apurinic/apyrimidinic lyase involved in base excision repair (Eide et al., 1996), was identified by DSSA as required for DMSO 
Table 2 | MIPS or GO categories associated with genes required for DMSO resistance.

\begin{tabular}{|c|c|c|c|c|}
\hline & $p$-value & Genes identified & $\boldsymbol{k}^{\mathbf{a}}$ & $f^{b}$ \\
\hline Cytoplasm to vacuole targeting (CVT) pathway [GO:0032258] & $2.38 \mathrm{E}-006$ & COG7 IRS4 COG8 COG6 COG5 & 5 & 37 \\
\hline \multicolumn{5}{|l|}{ GO CELLULAR COMPONENT CATEGORY } \\
\hline Golgi transport complex [GO:0017119] & 7.94E-008 & COG7 COG8 COG6 COG5 & 4 & 8 \\
\hline Intra Golgi transport [20.09.07.05] & 4.16E-005 & COG7 COG8 COG6 COG5 & 4 & 33 \\
\hline
\end{tabular}

Strains exhibiting sensitivity to $1 \%$ DMSO, as identified by DSSA, were analyzed with FunSpec for overrepresented biological attributes.

${ }^{a}$ Number of genes in category identified as sensitive to DMSO.

${ }^{b}$ Number of genes in GO or MIPS category.

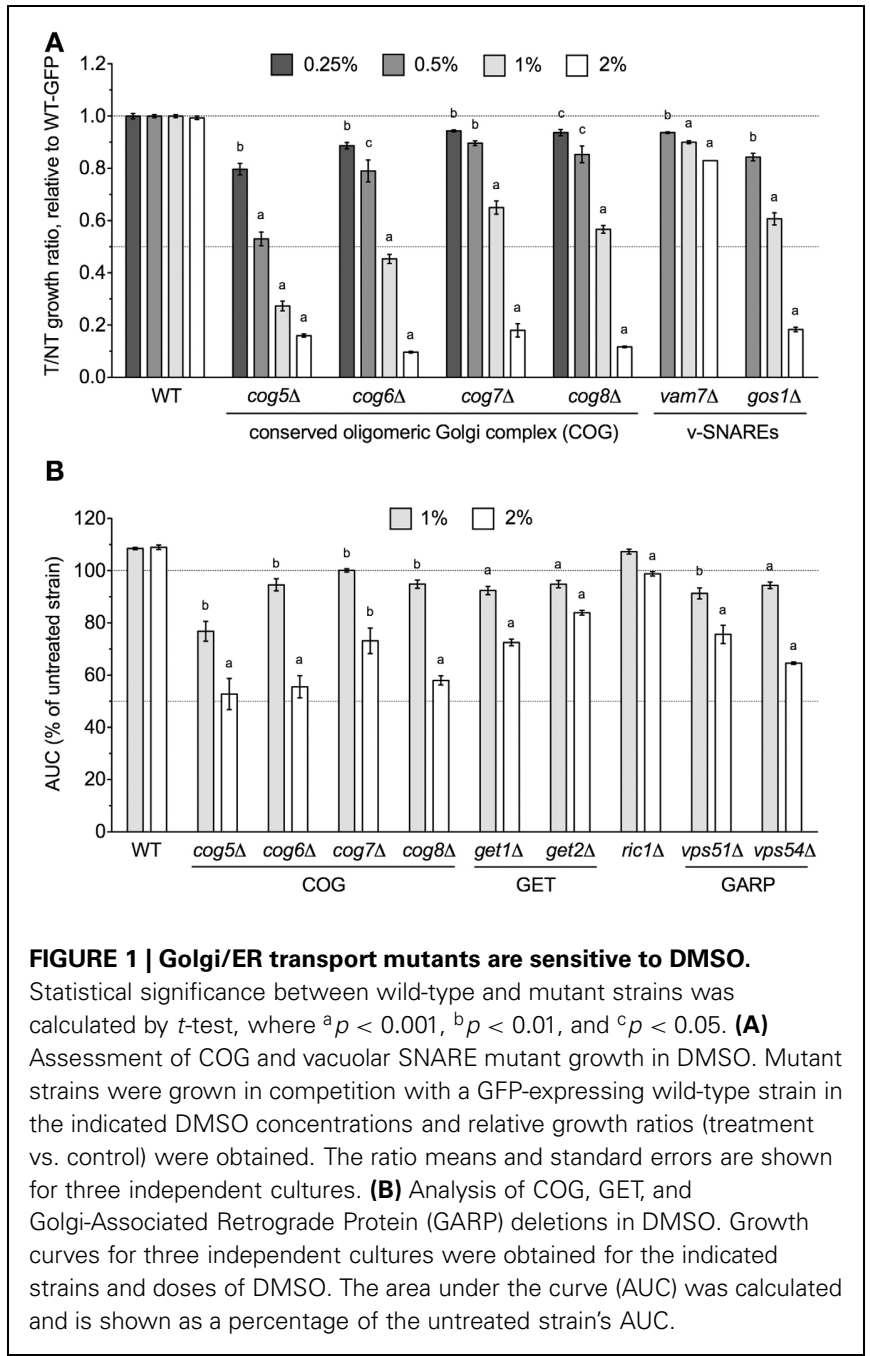

resistance (Table 1). Our relative growth assay confirmed $n \operatorname{tg} 1 \Delta$ as sensitive to DMSO, but interestingly, deletion of the NTG1 paralog NTG2 did not markedly alter growth in DMSO (Figure 4A). A strain deleted for MRE11, a component of the meiotic recombination (MRX) complex involved in repair of DNA double-strand breaks (and exhibiting synthetic sickness with EAF1 of NuA4), was also sensitive to DMSO (Figure 4A). Deletions in prefoldin (pac10 $\Delta$ and $y k e 2 \Delta$ ), a complex involved in the folding of tubulin and actin, were sensitive to DMSO (Figure 4B). Other genes necessary for DMSO tolerance included ROM2 (a GDP/GTP exchange factor for the Rho family), EDO1 (of unknown function), RRP8 (an rRNA methyltransferase), and KAP123 (a nuclear importer of histones $\mathrm{H} 3$ and H4) (Figure 4C).

\section{OVEREXPRESSION OF H2A.Z CONFERS RESISTANCE TO DMSO}

After demonstrating a role for the SWR1 histone exchange machinery and its accessories in DMSO tolerance (Figure 2), we examined whether overexpression of Htzlp (histone H2A.Z exchanged for $\mathrm{H} 2 \mathrm{~A}$ by SWR1) or Arp6p (the nucleosome binding component of SWR1) could rescue the DMSO sensitivity of various strains. Increased levels of Htzlp reversed the DMSO sensitivity of BY4743 wild-type and $h t z 1 \Delta$, but interestingly, caused growth defects with $1 \%$ DMSO in the yaf9s strain (Figure 5). It did not affect sensitivity of the $n t g 1 \Delta$ DNA repair mutant (data not shown). Although Arp6p overexpression provided DMSO resistance to the $n \operatorname{tg} 1 \Delta$ mutant (Figure 5), it did not alter the growth of wild-type, $h t z 1 \Delta$, or yaf9s strains in DMSO (data not shown).

\section{DISCUSSION}

DMSO is a polar and aprotic solvent commonly utilized to solubilize chemicals during toxicological or pharmaceutical inquiries (Santos et al., 2003). Compared to other solvents within its class such as sulfolane, $N, N$-dimethylformamide, $N$-methylpyrrolidin-2-one, or N,N-dimethyl acetamide, DMSO exhibits relatively limited acute toxicity (Tilstam, 2012), thus affording it preferred status within these fields. Despite its universality, DMSO's molecular mechanism(s) of action remain ambiguous, thus requiring investigations into the cellular processes and pathways it may perturb. Here we conducted a genome-wide functional screen in the model eukaryote $S$. cerevisiae to identify the non-essential yeast deletion mutants experiencing growth defects in $1 \%$ DMSO, a concentration typical to yeast toxicant or drug profiling studies. We demonstrate that components of the COG Golgi/ER transport and SWR1 histone exchange 

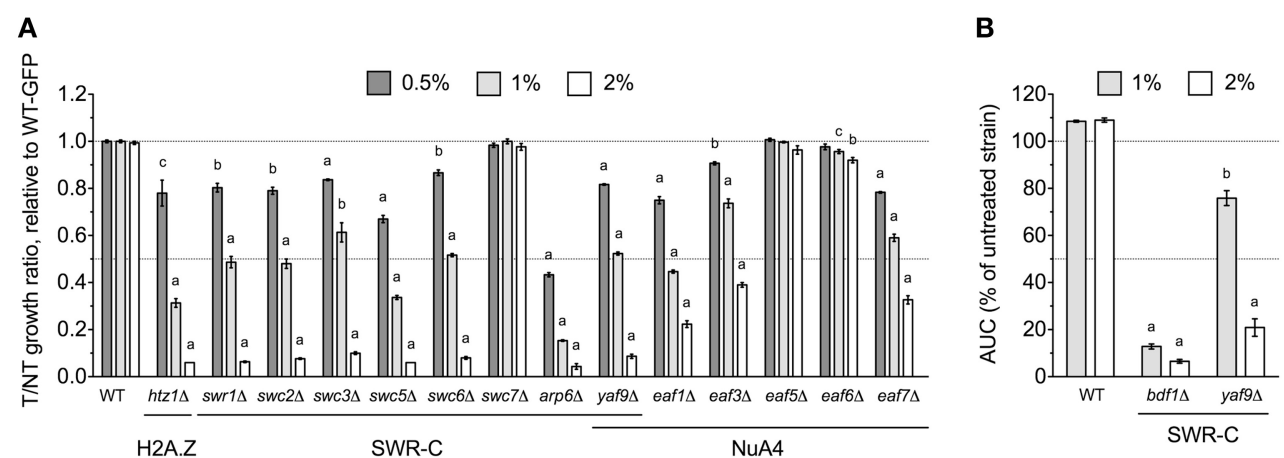

FIGURE 2 | SWR1 histone exchange and NuA4 histone H4 acetyltransferase mutants are sensitive to DMSO. Statistical significance between wild-type and mutant strains was determined by $t$-test, where ${ }^{a} p<0.001,{ }^{b} p<0.01$, and ${ }^{c} p<0.05$. (A) Assessment of DMSO treatment on strains lacking components of SWR1 or
NuA4. Relative growth ratios were obtained for three independent cultures and analyzed as described in Materials and Methods. (B) Evaluation of the bdf1s and yaf9s SWR1 mutants in DMSO.

Growth curves were acquired from three independent cultures at the indicated doses.
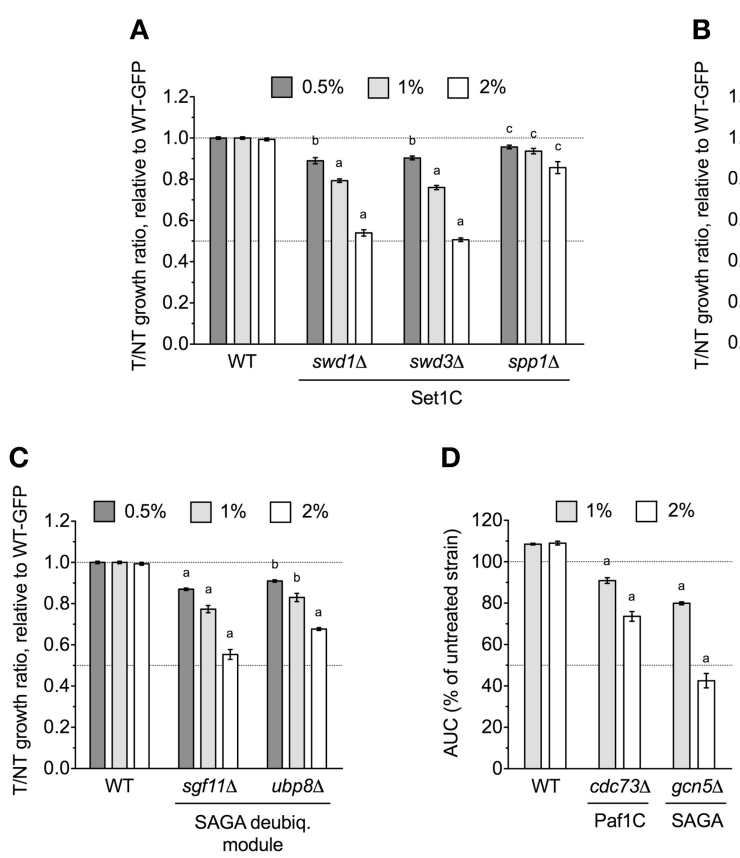
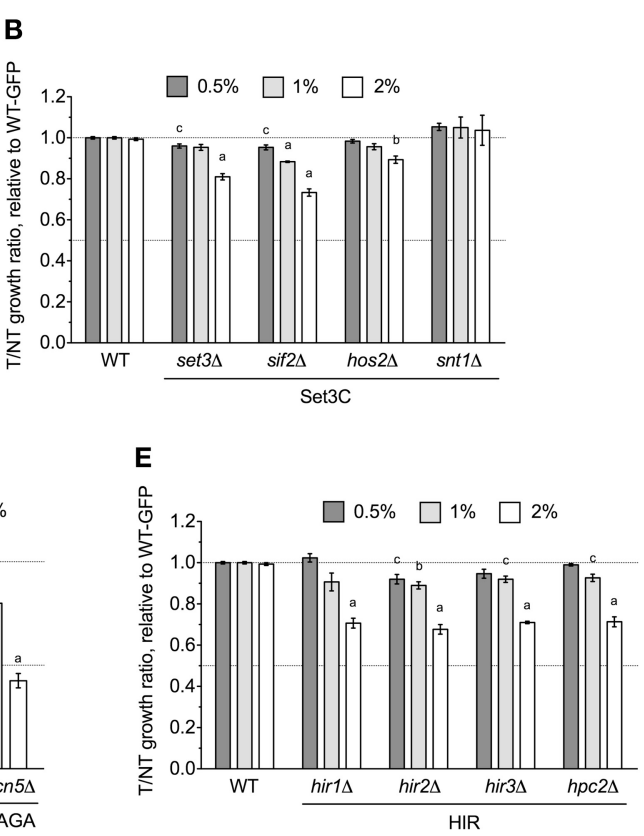

FIGURE 3 | Various chromatin remodeling mutants are sensitive to DMSO. Statistical significance between wild-type and mutant strains was calculated by $t$-test, with ${ }^{a} p<0.001$, ${ }^{b} p<0.01$, and ${ }^{c} p<0.05$. (A) Relative growth assays for Set1C histone $\mathrm{H} 3$ methylase mutants in DMSO. (B) Relative growth assays for DMSO-treated Set3C histone deacetylase mutants. (C) Evaluation of DMSO treatment on strains lacking SAGA histone H2B deubiquitylation module components. (D) Growth curves for SAGA and Paf1 mutants in DMSO. (E) Relative growth experiments for DMSO-exposed HIR mutants. For $(\mathbf{A}-\mathbf{C}, \mathbf{E})$, relative growth ratios were obtained and averaged for three independent cultures, while (D) displays average area under the curve data for growth curves acquired from three cultures. complexes are required for DMSO tolerance in yeast, with various mutants displaying sensitivity at concentrations as low as $0.25 \%$ (Figures 1, 2). Although many DMSO resistance genes are conserved in humans (Table 3), we were unable to confirm a role in DMSO tolerance for the COG5, NTG1, and YAF9 homologs in the nematode Caenorhabditis elegans or the COG7 and COG8 homologs in human fibroblasts (data not shown). These results may indicate that DMSO's mechanism of toxicity in yeast is different from that exhibited in nematodes or human cells. However, if the toxic mechanism remains similar, it is feasible that compensatory cellular processes or genes are present in these mutants.

During the preparation of this manuscript, a report was published describing functional profiling of yeast mutants in DMSO (Zhang et al., 2013), with findings congruent to those presented in this study (see Table 4 for a comparison of strains identified). In this section, we discuss various aspects differentiating our investigation from Zhang et al. (2013). First, while 

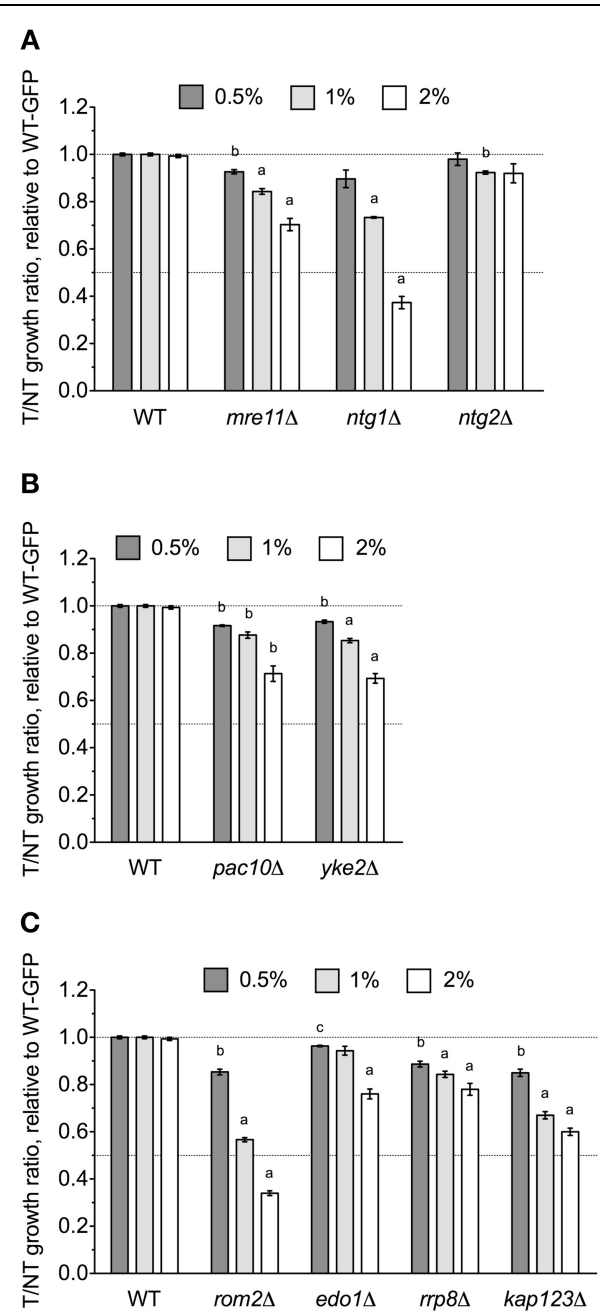

FIGURE 4 | DNA repair and other various mutants are sensitive to DMSO. Relative growth assays were performed for three independent cultures. Ratio means and standard errors are shown, with statistical significance between wild-type and mutant strains calculated by $t$-test, where ${ }^{a} p<0.001,{ }^{b} p<0.01$, and ${ }^{c} p<0.05$. (A) Analysis of DNA repair mutant growth in DMSO. (B) Relative growth assays in DMSO with mutants lacking prefoldin components. (C) A summary of various additional mutants tested for sensitivity to DMSO.

these researchers assessed growth of individual yeast mutants via colony size on solid media, we performed functional profiling in pooled liquid cultures under competitive growth conditions. Our analyses, in which DNA sequences unique to each strain are hybridized to a microarray after toxicant exposure, are able to discern small growth defects and can identify sensitive strains overlooked by other methods (Table 4). However, the stringency of our DSSA may hinder identification of slow growing strains or those close to background levels. Nevertheless, these data are extremely relevant to those conducting pooled growth assays, especially considering the increased popularity of automated screens and high-throughput multiplexed barcode sequencing to examine strain growth in DMSO-soluble toxicants or drugs

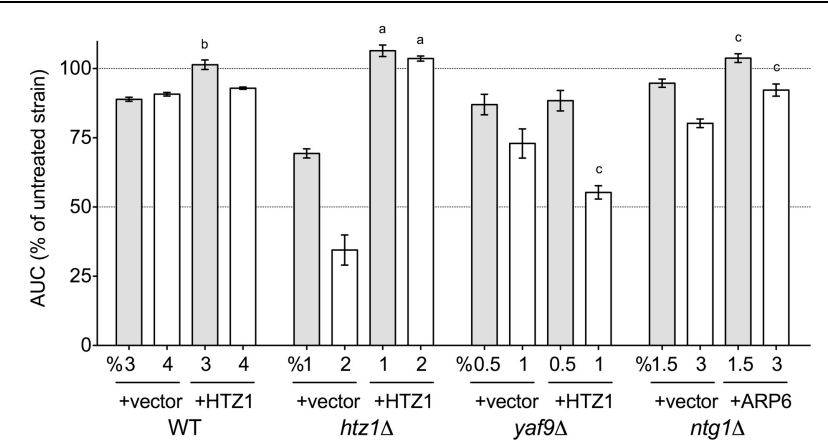

FIGURE 5 | Overexpression of Htz1p or Arp6p rescues DMSO sensitivity in various mutants. Growth curves for three independent cultures were obtained in the indicated doses of DMSO. The area under the curve (AUC) means and standard error are shown. Statistical significance between AUCs for corresponding doses in the empty vector and overexpression strains was calculated by $t$-test, and is indicated by ${ }^{\mathrm{a}} p<0.001,{ }^{\mathrm{b}} p<0.01$, and ${ }^{\mathrm{c}} p<0.05$.

(Smith et al., 2010, 2012). Second, compared to the use of 4 and $8 \%$ DMSO in Zhang et al. (2013), the concentrations utilized in our screen (1\%) and confirmation assays (0.25-2\%) do not inhibit growth of the BY4743 wild-type strain and represent levels standard to functional screens ( $1 \%$ or less). The contrasting choice of doses may also account for differences in the DMSO-sensitive strains identified by each screen. Third, we provide extensive DMSO dose-response analyses for novel DMSOsensitive strains as well as those concomitantly identified by Zhang et al. (2013). Finally, our overexpression data demonstrates that increased levels of Htzlp or Arp6p can rescue the growth of various deletion strains in DMSO (Figure 5).

We have identified three cellular processes influencing DMSO resistance in budding yeast: Golgi/ER trafficking, SWR1 complex action, and DNA repair. Microarray analyses assessing the response of S. cerevisiae to DMSO (Zhang et al., 2003) did not identify any genes described in this study, however, correlation between transcriptional events and genes required for growth under a selective condition is often low (Giaever et al., 2002). The requirement of COG and SNARE Golgi/ER genes for DMSO tolerance (Figure 1) may reflect findings in human and rat hepatocytes, where DMSO altered expression of genes associated with SNARE interactions in vesicular transport (Sumida et al., 2011). Furthermore, as a "chemical chaperone," DMSO can mimic the function of molecular chaperones (Papp and Csermely, 2006), a group of proteins closely tied to Golgi/ER operations. The DMSO sensitivity of histone H2A.Z and chromatin remodeling mutants (Figures 2, 3) indicate DMSO may affect chromatin structure. Lapeyre and Bekhor (1974) reported that 1\% DMSO decreased chromatin thermostability, while higher concentrations promoted chromatin relaxation. Consistent with these findings, Pommier et al. (1983) suggested DMSO increased domain (loop) size by reducing DNA-protein attachment points after finding it enhanced intercalator-induced DNA breakage. DMSO could conceivably cause DNA damage, as demonstrated by DNA repair mutant sensitivity (Figure 4A). DMSO damaged DNA in bull sperm (Taşdemir et al., 2013) and erythroleukemic cells (Scher 
Table 3 | Human orthologs of yeast genes required for DMSO tolerance.

\begin{tabular}{|c|c|c|}
\hline Yeast gene & $\begin{array}{l}\text { Human } \\
\text { ortholog(s) }\end{array}$ & Human protein description \\
\hline ARP6 & ACTR6 & ARP6 actin-related protein 6 homolog \\
\hline$B D F 1$ & EP300 & Histone acetyltransferase \\
\hline$C D C 73$ & $C D C 73$ & $\begin{array}{l}\text { Component of the PAF1 complex; tumor } \\
\text { suppressor }\end{array}$ \\
\hline COG5 & COG5 & Component of oligomeric Golgi complex 5 \\
\hline COG6 & COG6 & Component of oligomeric Golgi complex 6 \\
\hline COG7 & COG7 & Component of oligomeric Golgi complex 7 \\
\hline COG8 & COG8 & component of oligomeric Golgi complex 8 \\
\hline EAF3 & MORF4L1 & $\begin{array}{l}\text { Component of the NuA4 histone } \\
\text { acetyltransferase complex }\end{array}$ \\
\hline EAF6 & MEAF6 & $\begin{array}{l}\text { Component of the NuA4 histone } \\
\text { acetyltransferase complex }\end{array}$ \\
\hline EAF7 & $M R G B P$ & $\begin{array}{l}\text { Component of the NuA4 histone } \\
\text { acetyltransferase complex }\end{array}$ \\
\hline GCN5 & KAT2A & Histone acetyltransferase \\
\hline GOS1 & GOSR1 & $\begin{array}{l}\text { Involved in ER-Golgi transport as well as } \\
\text { intra-Golgi transport }\end{array}$ \\
\hline$H I R 1 / 2$ & HIRA & Histone chaperone \\
\hline HOS2 & HDAC3 & Histone deacetylase \\
\hline HTZ1 & $H 2 A F Z$ & $\begin{array}{l}\text { Variant histone } \mathrm{H} 2 \mathrm{~A} \text {; replaces conventional } \\
\mathrm{H} 2 \mathrm{~A} \text { in a subset of nucleosomes }\end{array}$ \\
\hline KAP123 & IPO4 & Nuclear transport receptor \\
\hline MRE11 & MRE11A & $\begin{array}{l}\text { Component of MRN complex; involved in } \\
\text { DNA double-strand break repair }\end{array}$ \\
\hline NTG1 & NTHL 1 & $\begin{array}{l}\text { Apurinic and/or apyrimidinic endonuclease } \\
\text { and DNA N-glycosylase }\end{array}$ \\
\hline PAC10 & $V B P 1$ & $\begin{array}{l}\text { Transfers target proteins to cytosolic } \\
\text { chaperonin }\end{array}$ \\
\hline RRP8 & $R R P 8$ & $\begin{array}{l}\text { Component of the eNoSC complex; } \\
\text { mediates silencing of rDNA }\end{array}$ \\
\hline SIF2 & TBLIX & $\begin{array}{l}\text { Subunit in corepressor SMRT complex } \\
\text { along with HDAC3 }\end{array}$ \\
\hline SPP1 & $C X X C 1$ & $\begin{array}{l}\text { Recognizes } \mathrm{CpG} \text { sequences and regulates } \\
\text { gene expression }\end{array}$ \\
\hline SWC2 & VPS72 & $\begin{array}{l}\text { subunit of acetyltransferase TRRAP/TIP60 } \\
\text { and chromatin-remodeling SRCAP }\end{array}$ \\
\hline SWC5 & CFDP1 & $\begin{array}{l}\text { Craniofacial development protein } 1 \text {; may } \\
\text { play role in embryogenesis }\end{array}$ \\
\hline SWC6 & ZNHIT1 & Zinc finger, HIT-type containing 1 \\
\hline SWD1 & RBBP5 & $\begin{array}{l}\text { Component of MLL1/MLL histone } \\
\text { methyltransferase complex }\end{array}$ \\
\hline SWD3 & WDR5 & $\begin{array}{l}\text { Component of MLL1/MLL histone } \\
\text { methyltransferase complex }\end{array}$ \\
\hline SWR1 & SRCAP & $\begin{array}{l}\text { Catalytic component of the } \\
\text { chromatin-remodeling SRCAP complex }\end{array}$ \\
\hline UBP8 & USP22 & $\begin{array}{l}\text { Histone deubiquitinating component of } \\
\text { SAGA histone acetylation complex }\end{array}$ \\
\hline VAM7 & SNAP25 & $\begin{array}{l}\text { t-SNARE involved in the molecular } \\
\text { regulation of neurotransmitter release }\end{array}$ \\
\hline VPS51 & VPS51 & $\begin{array}{l}\text { Required for both Golgi structure and } \\
\text { vesicular trafficking }\end{array}$ \\
\hline VPS54 & VPS54 & $\begin{array}{l}\text { Required for retrograde transport of } \\
\text { proteins from prevacuoles to the late Golgi }\end{array}$ \\
\hline YAFO & YEATS4 & $\begin{array}{l}\text { Component of the NuA4 histone } \\
\text { acetyltransferase complex }\end{array}$ \\
\hline YKE2 & PFDN6 & $\begin{array}{l}\text { Subunit of heteromeric prefoldin; transfers } \\
\text { proteins to cytosolic chaperonin }\end{array}$ \\
\hline
\end{tabular}

Deletion of the yeast genes listed resulted in sensitivity to DMSO (shown in alphabetical order).
Table 4 | A comparison between studies identifying yeast genes responsible for DMSO tolerance.

\begin{tabular}{|c|c|c|c|c|}
\hline \multicolumn{2}{|c|}{$\begin{array}{l}\text { DMSO tolerance genes } \\
\text { identified by Zhang et al. (2013) } \\
\text { and this study }\end{array}$} & \multicolumn{3}{|c|}{$\begin{array}{l}\text { DMSO tolerance genes } \\
\text { identified by this } \\
\text { study }\end{array}$} \\
\hline$A R P 6$ & ROM2 & COG5 & KAP123 & $U B C 8$ \\
\hline$B D F 1$ & SET3 & COG8 & MRE11 & VPS54 \\
\hline$C D C 73$ & SWC2 (VPS72) & EAF6 & NTG1 & YAF9 \\
\hline COG6 & SWC3 & EAF7 & PAC10 & YKE2 \\
\hline COG7 & SWC6 (VPS71) & EDO1 & $R / C 1$ & \\
\hline EAF1 & SWC7 & GCN5 & $R R P 8$ & \\
\hline EAF3 & SWD1 & GET1 & SGF11 & \\
\hline GOS1 & SWR1 & GET2 & SIF2 & \\
\hline HIR2 & VAM7 & $H I R 1$ & SPP1 & \\
\hline HOS2 & VPS51 & HIR3 & SWC5 & \\
\hline HTZ1 & & HPC2 & SWD3 & \\
\hline
\end{tabular}

DMSO tolerance genes identified by Zhang et al. (2013) were compared to those identified in this study.

and Friend, 1978), and additionally altered expression of DNA repair genes in human and rat hepatocytes (Sumida et al., 2011).

The experimental evidence integrating the seemingly discrete processes of Golgi/ER transport, SWR1 complex action, and DNA repair is limited. Strains lacking SWR1 and NuA4 components exhibit synthetic lethality or sickness with various Golgi/ER transport and DNA repair genes (Collins et al., 2007; Mitchell et al., 2008; Costanzo et al., 2010; Hoppins et al., 2011), but mechanistic data explaining these findings are lacking. If Golgi/ER transport is the crucial determinant of DMSO tolerance, it is reasonable that loss of SWR1, which may repress transcription by preventing histone H2A.Z deposition into chromatin (Meneghini et al., 2003; Zhang et al., 2005), could confer DMSO sensitivity by decreasing production of Golgi/ER transport genes. Expression of COG7, a COG member involved in Golgi/ER trafficking, is downregulated in $h t z 1 \Delta$ and the SWR1 mutants $\operatorname{swr} 1 \Delta, \operatorname{swc} 2 \Delta$, and $\operatorname{swc5} \Delta$ (Morillo-Huesca et al., 2010), but others report the nonessential COG genes are neither induced nor repressed in the swr1 $\Delta$ background (Meneghini et al., 2003). Alternatively, if SWR1 or H2A.Z activity is the deciding factor in DMSO resistance, defective Golgi/ER transport could prevent appropriate processing and localization of SWR1 components or H2A.Z. However, the expression of Golgi/ER, chromatin remodeling, or DNA repair genes described herein are not altered in $h t z 1 \Delta$, and further, HTZ1 expression is unchanged in SWR1 or NuA4 mutants (Meneghini et al., 2003; Lindstrom et al., 2006; Lenstra et al., 2011). The relationship of SWR1 to DNA repair is evidenced by its ability to cause genetic instability in the absence of H2A.Z (Morillo-Huesca et al., 2010) and also deposit H2A.Z at double-stranded DNA breaks (Kalocsay et al., 2009).

We provide valuable insight into the genetic requirements for DMSO tolerance by identifying three major cellular processesGolgi/ER transport, SWR1 complex function, and DNA repairas important in DMSO resistance in S. cerevisiae. To separate effects of DMSO from a compound of interest, it is crucial for future yeast profiling studies to recognize that various deletion strains may fall out of pooled cultures during treatment with 
DMSO-solubilized drugs or toxicants. Data gathered by our study can direct additional experimentation to decipher the cellular and molecular mechanisms of DMSO action.

\section{ACKNOWLEDGMENTS}

This work was supported by the National Institute of Environmental Health Sciences Superfund Research Program [grant number P42ES004705 awarded to Martyn T. Smith, Chris D. Vulpe is leader on Project 2]. The content is solely the responsibility of the authors and does not represent the official views of the funding agencies. We thank Aaron Welch of the Koshland laboratory (University of California, Berkeley) for the HTZ1 and ARP6 HIP FlexGenes and Mani Tagmount of the Vulpe laboratory (University of California, Berkeley) for helpful discussions. C. elegans strains were provided by the Caenorhabditis Genetics Center (CGC), funded by NIH Office of Research
Infrastructure Programs (P40 OD010440). The GM0038 control and COG8 mutant fibroblasts were a gift from Hudson Freeze, while the COG7 mutant fibroblasts were a gift from Richard Steet. Brandon D. Gaytán is a trainee in the Superfund Research Program at the University of California, Berkeley. Conceived and designed the experiments: Brandon D. Gaytán, Vanessa Y. De La Rosa, and Chris D. Vulpe. Performed the experiments: Brandon D. Gaytán and Jan-Michael Lerot. Analyzed the data: Brandon D. Gaytán, Alex V. Loguinov, Vanessa Y. De La Rosa, and Chris D. Vulpe. Wrote the paper: Brandon D. Gaytán and Chris D. Vulpe.

\section{SUPPLEMENTARY MATERIAL}

The Supplementary Material for this article can be found online at: http://www.frontiersin.org/Toxicogenomics_/ 10.3389/fgene.2013.00154/abstract

\section{REFERENCES}

Blackman, R. K., Cheung-Ong, K., Gebbia, M., Proia, D. A., He, S., Kepros, J., et al. (2012). Mitochondrial electron transport is the cellular target of the oncology drug elesclomol. PLoS ONE 7:e29798. doi: 10.1371/journal.pone.0029798

Collins, S. R., Miller, K. M., Maas, N. L., Roguev, A., Fillingham, J., Chu, C. S., et al. (2007). Functional dissection of protein complexes involved in yeast chromosome biology using a genetic interaction map. Nature 446, 806-810. doi: 10.1038/nature05649

Costanzo, M., Baryshnikova, A., Bellay, J., Kim, Y., Spear, E. D., Sevier, C. S., et al. (2010). The genetic landscape of a cell. Science 327, 425-431. doi: 10.1126/science. 1180823

Eide, L., Bjørås, M., Pirovano, M., Alseth, I., Berdal, K. G., and Seeberg, E. (1996). Base excision of oxidative purine and pyrimidine DNA damage in Saccharomyces cerevisiae by a DNA glycosylase with sequence similarity to endonuclease III from Escherichia coli. Proc. Natl. Acad. Sci. U.S.A. 93, 10735-10740. doi: 10.1073/pnas.93. 20.10735

Gaytán, B. D., Loguinov, A. V., Lantz, S. R., Lerot, J.-M., Denslow, N. D., and Vulpe, C. D. (2013). Functional profiling discovers the dieldrin organochlorinated pesticide affects leucine availability in yeast. Toxicol. Sci. 132, 347-358. doi: 10.1093/toxsci/kft018

Giaever, G., Chu, A. M., Ni, L., Connelly, C., Riles, L., Véronneau, S., et al. (2002). Functional profiling of the Saccharomyces cerevisiae genome. Nature 418, 387-391. doi: 10.1038/nature00935
Hoppins, S., Collins, S. R., CassidyStone, A., Hummel, E., Devay, R. M., Lackner, L. L., et al. (2011). A mitochondrial-focused genetic interaction map reveals a scaffoldlike complex required for inner membrane organization in mitochondria. J. Cell Biol. 195, 323-340. doi: $10.1083 /$ jcb. 201107053

Jo, W. J., Ren, X., Chu, F., Aleshin, M., Wintz, H., Burlingame, A., et al. (2009a). Acetylated H4K16 by MYST1 protects UROtsa cells from arsenic toxicity and is decreased following chronic arsenic exposure. Toxicol. Appl. Pharmacol. 241, 294-302. doi: 10.1016/j.taap.2009.08.027

Jo, W. J., Loguinov, A., Wintz, H., Chang, M., Smith, A. H., Kalman, D., et al. (2009b). Comparative functional genomic analysis identifies distinct and overlapping sets of genes required for resistance to monomethylarsonous acid (MMAIII) and arsenite (AsIII) in yeast. Toxicol. Sci. 111, 424-436. doi: 10.1093/toxsci/kfp162

Kalocsay, M., Hiller, N. J., and Jentsch, S. (2009). Chromosome-wide Rad51 spreading and SUMOH2A.Z-dependent chromosome fixation in response to a persistent DNA double-strand break. Mol. Cell 33, 335-343. doi: 10.1016/j.molcel.2009.01.016

Lapeyre, J.-N., and Bekhor, I. (1974). Effects of 5-bromo-2'-deoxyuridine and dimethyl sulfoxide on properties and structure of chromatin. J. Mol. Biol. 89, 137-162. doi 10.1016/0022-283690167-3

Lenstra, T. L., Benschop, J. J., Kim, T., Schulze, J. M., Brabers, N. A. C. H., Margaritis, T., et al. (2011). The specificity and topology of chromatin interaction pathways in yeast. Mol. Cell 42, 536-549. doi 10.1016/j.molcel.2011.03.026

Lindstrom, K. C., Vary, J. C., Parthun, M. R., Delrow, J., and Tsukiyama, T. (2006). Isw1 functions in parallel with the NuA4 and Swr 1 complexes in stress-induced gene repression. Mol. Cell Biol. 26, 6117-6129. doi: 10.1128/MCB.00642-06

Lu, P. Y. T., Lévesque, N., and Kobor, M. S. (2009). NuA4 and SWR1C: two chromatin-modifying complexes with overlapping functions and components. Biochem. Cell Biol. 87, 799-815. doi: 10.1139/O09-062

Meneghini, M. D., Wu, M., and Madhani, H. D. (2003). Conserved histone variant H2A.Z protects euchromatin from the ectopic spread of silent heterochromatin. Cell 112, 725-736. doi 10.1016/S0092-867400123-5

Mitchell, L., Lambert, J.-P., Gerdes, M., Al-Madhoun, A. S., Skerjanc, I. S., Figeys, D., et al. (2008). Functional dissection of the NuA4 histone acetyltransferase reveals its role as a genetic hub and that Eafl is essential for complex integrity. Mol. Cell Biol. 28, 2244-2256. doi 10.1128/MCB.01653-07

Mizuguchi, G., Shen, X., Landry, J., Wu, W.-H., Sen, S., and Wu, C. (2004). ATP-driven exchange of histone $\mathrm{H} 2 \mathrm{AZ}$ variant catalyzed by SWR1 chromatin remodeling complex. Science 303, 343-348. doi: 10.1126/science. 1090701

Morillo-Huesca, M., Clemente-Ruiz, M., Andújar, E., and Prado, F. (2010). The SWR1 histone replacement complex causes genetic instability and genome-wide transcription misregulation in the absence of H2A.Z. PLoS ONE 5:e12143. doi: 10.1371/journal. pone. 0012143
North, M., Tandon, V. J., Thomas, R., Loguinov, A., Gerlovina, I., Hubbard, A. E., et al. (2011). Genome-wide functional profiling reveals genes required for tolerance to benzene metabolites in yeast. PLOS ONE 6:e24205. doi: 10.1371/ journal.pone.0024205

Papp, E., and Csermely, P. (2006) Chemical chaperones: mechanisms of action and potential use. Handb. Exp. Pharmacol. 172, 405-416. doi: 10.1007/3-540-29717-0_16

Pommier, Y., Zwelling, L. A., Mattern, M. R., Erickson, L. C., Kerrigan, D., Schwartz, R., et al. (1983). Effects of dimethyl sulfoxide and thiourea upon intercalator-induced DNA single-strand breaks in mouse leukemia (L1210) cells. Cancer Res. 43, 5718-5724.

Robinson, M., Grigull, J., Mohammad, N., and Hughes, T. (2002). FunSpec: a web-based cluster interpreter for yeast. BMC Bioinformatics 3:35. doi: 10.1186/1471-2105-3-35

Santos, N. C., Figueira-Coelho, J., Martins-Silva, J., and Saldanha, C. (2003). Multidisciplinary utilization of dimethyl sulfoxide: pharmacological, cellular, and molecular aspects. Biochem. Pharmacol. 65, 1035-1041. doi 10.1016/S0006-295200002-9

Scher, W., and Friend, C. (1978). Breakage of DNA and alterations in folded genomes by inducers of differentiation in friend erythroleukemic cells. Cancer Res. 38, 841-849.

Smith, A. M., Durbic, T., Kittanakom, S., Giaever, G., and Nislow, C. (2012). Barcode sequencing for understanding drug-gene interactions. Methods Mol. Biol. 910, 55-69. doi: 10.1007/978-1-61779965-5_4 
Smith, A. M., Heisler, L. E., St. Onge, R. P., Farias-Hesson, E., Wallace, I. M., Bodeau, J., et al. (2010). Highlymultiplexed barcode sequencing: an efficient method for parallel analysis of pooled samples. Nucleic Acids Res. 38:e142. doi: 10.1093/nar/gkq368

Steinmetz, L. M., Scharfe, C., Deutschbauer, A. M., Mokranjac, D., Herman, Z. S., Jones, T., et al. (2002). Systematic screen for human disease genes in yeast. Nat. Genet. 31, 400-404.

Sumida, K., Igarashi, Y., Toritsuka, N., Matsushita, T., Abe-Tomizawa, K., Aoki, M., et al. (2011). Effects of DMSO on gene expression in human and rat hepatocytes. Hum. Exp. Toxicol. 30, 1701-1709. doi: 10.1177/0960327111399325

Taşdemir, U., Büyükleblebici, S., Tuncer, P. B., Coşkun, E., Özgürtaş, T., Ayd $\iota$ n, F. N., et al. (2013). Effects of various cryoprotectants on bull sperm quality, DNA integrity and oxidative stress parameters. Cryobiology 66, 38-42. doi: 10.1016/ j.cryobiol.2012.10.006

Tilstam, U. (2012). Sulfolane: A versatile dipolar aprotic solvent. Org. Process Res. Dev. 16, 1273-1278. doi: 10.1021/op300108w

Yu, Z. W., and Quinn, P. J. (1994). Dimethyl sulphoxide: a review of its applications in cell biology. Biosci. Rep. 14, 259-281. doi: $10.1007 /$ BF01199051

Zhang, H., Roberts, D. N., and Cairns, B. R. (2005). Genome-wide dynamics of Htz1, a histone H2A variant that poises repressed/basal promoters for activation through histone loss. Cell 123, 219-231. doi: 10.1016/j.cell.2005.08.036

Zhang, L., Liu, N., Ma, X., and Jiang, L. (2013). The transcriptional control machinery as well as the cell wall integrity and its regulation are involved in the detoxification of the organic solvent dimethyl sulfoxide in Saccharomyces cerevisiae. FEMS Yeast Res. 13, 200-218. doi: 10.1111/ 1567-1364.12022

Zhang, W., Needham, D. L., Coffin, M., Rooker, A., Hurban, P., Tanzer, M. M., et al. (2003). Microarray analyses of the metabolic responses of Saccharomyces cerevisiae to organic solvent dimethyl sulfoxide. J. Ind. Microbiol. Biotechnol. 30, 57-69.

Conflict of Interest Statement: The authors declare that the research was conducted in the absence of any commercial or financial relationships that could be construed as a potential conflict of interest.

Received: 18 June 2013; paper pending published: 12 July 2013; accepted: 24 July 2013; published online: 13 August 2013.
Citation: Gaytán BD, Loguinov AV, De La Rosa VY, Lerot J-M and Vulpe CD (2013) Functional genomics indicates yeast requires Golgi/ER transport, chromatin remodeling, and DNA repair for low dose DMSO tolerance. Front. Genet. 4:154. doi: 10.3389/fgene.2013.00154

This article was submitted to Frontiers in Toxicogenomics, a specialty of Frontiers in Genetics.

Copyright (c) 2013 Gaytán, Loguinov, De La Rosa, Lerot and Vulpe. This is an open-access article distributed under the terms of the Creative Commons Attribution License (CC BY). The use, distribution or reproduction in other forums is permitted, provided the original author(s) or licensor are credited and that the original publication in this journal is cited, in accordance with accepted academic practice. No use, distribution or reproduction is permitted which does not comply with these terms. 